\title{
Electron Diffraction Tomography and Dynamical Refinement for Crystal-Structure Characterization of Nanocrystalline Materials
}

\author{
C.A. CorrêA ${ }^{a, b, *}$, M. Klementová ${ }^{b}$ And L. Palatinus ${ }^{b}$
}

${ }^{a}$ Department of Physics of Materials, Charles University, Prague, Czech Republic

${ }^{b}$ Institute of Physics of the Academy of Sciences of the Czech Republic, Prague, Czech Republic

Three-dimensional electron diffraction tomography allows one to obtain structure information from nanocrystals. However, in order to get accurate results the dynamical theory must be used due to the strong dynamical interaction between electrons and matter. Full structure refinement using dynamical theory has been in use for some time, in spite of being hampered by the fact that the intensities are very sensitive to variations of thickness and of the orientation of the sample. A remedy to this problem is the technique called precession electron diffraction. The use of precession electron diffraction in combination with electron diffraction tomography results in more accurate structure parameters and lower figures of merit in the structure refinement. The principles of electron diffraction tomography, precession electron diffraction and dynamical refinement will be demonstrated on the structural analysis of a nanowire of $\mathrm{Ni}_{3} \mathrm{Si}_{2}$.

DOI: $10.12693 /$ APhysPolA.128.651

PACS: 61.05.J-, 61.66.-f, 61.46.Km

\section{Introduction}

The interest in developing nanodevices and nanotechnology demands accurate structure determination of nanocrystals, because of the dependence of the properties of the material on its structure. The technique commonly used for structure determination is single crystal X-ray diffraction, which needs crystals larger than $\approx 10 \mu \mathrm{m}$. Nevertheless, it is also possible to analyze structure of nanocrystals using electron diffraction (ED), because the interaction between electrons and matter is much stronger compared to X-rays.

Detailed three-dimensional structural information from nanocrystals can be obtained with electron diffraction tomography (EDT), where the sample is tilted in small steps along a fixed rotation axis and, at each step, a diffraction pattern is recorded. Since the positional angle of each diffraction pattern is known, the $3 \mathrm{D}$ information about the reciprocal space of the nanocrystal can be reconstructed $[1,2]$.

However, the strong interaction between electrons and matter causes multiple scattering within the sample. The kinematical approximation, which assumes that the electron is scattered only once when going through the material and which can be used for X-rays, is not valid for electron diffraction. If kinematical approximation is used, it results in distorted structures with unreliable structure parameters. For more accurate and more reliable results, the dynamical theory of diffraction must be used. The dynamical theory takes into account multiple scattering of the electron beam when passing through the crystal.

*corresponding author; e-mail: cinthiacac@gmail.com
During the structure refinement using dynamical theory, the dependence of the intensities on thickness and orientation is diminished by the use of the precession electron diffraction (PED), developed by Vincent and Midgley [3]. In the PED the incident electron beam is deflected from the optical axis of the transmission electron microscope (TEM) by a few degrees, is precessed around the optical axis of the TEM and is re-deflected by the coils under the specimen (Fig. 1). The diffracted intensities are integrated over all orientations of the beam during the precession. As a consequence, the intensities are less sensitive to imperfections of the crystal and more sensitive to the structural parameters like atomic positions $[4,5]$.

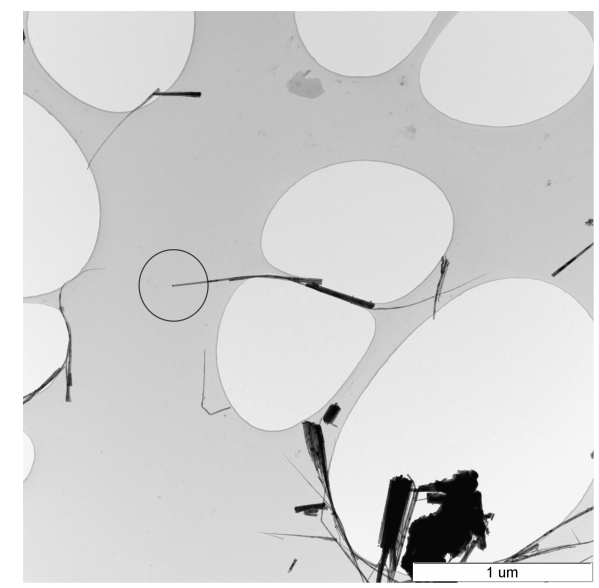

Fig. 1. Scheme of the PED, showing the incident beam precessed, forming a cone around the optical axis of the TEM. The coils under the sample re-deflect the beam to the optical axis, resulting in diffracted spots.

The use of PED in combination with EDT results in reliable structure parameters. The accuracy of the 
structure model is significantly improved when the dynamical theory is used for structure refinement of such data sets. To our knowledge, the only software using the dynamical approach for data sets collected combining PED and EDT is the crystallographic computing system JANA2006 [6, 7], developed in our laboratory.

In order to compare the kinematical approximation and the dynamical theory in the refinement of a data set collected using PED and EDT, the structure of a nanowire of $\mathrm{Ni}_{3} \mathrm{Si}_{2}$ was analyzed. Transition metal silicides are of great interest for use in micro- and nanodevices due to their properties, such as low electrical resistance and high-temperature stability [8].

\section{Experimental}

The nanowires were obtained by chemical vapor deposition (CVD) at the Institute of Chemical Processes Fundamentals of the AS CR, v.v.i. Nanocrystals were deposited on a copper grid with holey carbon film. The selected crystal had a diameter of $25 \mathrm{~nm}$.

The diffraction patterns were collected on the TEM PHILIPS CM120 with a $\mathrm{LaB}_{6}$ cathode at $120 \mathrm{kV}$, equipped with a precession device Nanomegas Digistar. The patterns were recorded with a CCD camera Olympus Veleta. The patterns were collected using selected area electron diffraction (SAED) with aperture of $40 \mu \mathrm{m}$ in order to have reflections from a smaller part of the crystal, which is represented by the circle in Fig. 2 .

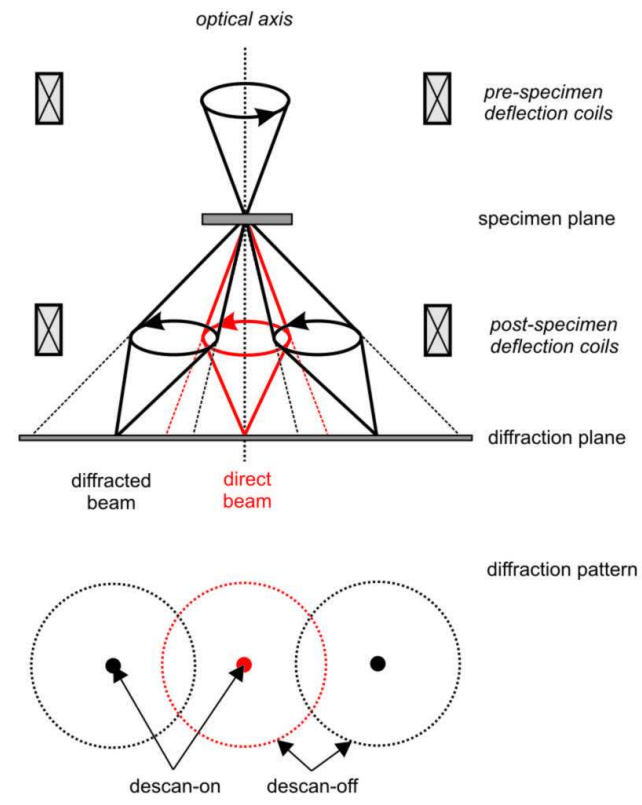

Fig. 2. Nanowire with the diameter of $25 \mathrm{~nm}$ used for the data collection. A smaller part of the crystal (circled in the picture) was used for the measurement with the use of selected area aperture.

EDT data were collected with tilt angle varying in steps of $1^{\circ}$ around the main axis of the sample holder, from $-52^{\circ}$ to $50^{\circ}$. The precession semi-angle was set to $\varphi=1^{\circ}$. The data were processed using the data analysis software PETS [9] and the unit cell was identified in the graphical interface of JANA2006. The structure solution was performed with Superflip [10], interfaced from JANA2006. The structure refinement was also performed in JANA2006. The kinematical and dynamical refinements were compared through the figures of merit $R$ (Eq. (1)), $w R$ (Eq. (2)) and GOF (Eq. (3)), and through the atomic coordinates. The figures of merit are defined as:

$$
\begin{aligned}
& R=\frac{\sum\left|\left(I_{\mathrm{g}}^{\circ}\right)^{1 / 2}-\left(I_{\mathrm{g}}^{c}\right)^{1 / 2}\right|}{\sum\left(I_{\mathrm{g}}^{\circ}\right)^{1 / 2}} \\
& w R=\left[\frac{\sum w_{\mathrm{g}}\left(I_{\mathrm{g}}^{\circ}-I_{\mathrm{g}}^{c}\right)^{2}}{\sum w_{\mathrm{g}}\left(I_{\mathrm{g}}^{\circ}\right)^{2}}\right]^{1 / 2}
\end{aligned}
$$

and

$$
w R=\left[\frac{\sum w_{\mathrm{g}}\left(I_{\mathrm{g}}^{\circ}-I_{\mathrm{g}}^{c}\right)^{2}}{\left(N_{\mathrm{R}}-N_{\mathrm{P}}\right)}\right]^{1 / 2},
$$

where $I_{\mathrm{g}}^{\circ}$ and $I_{\mathrm{g}}^{c}$ are the observed and calculated intensities of the beam $\boldsymbol{g}$, respectively, $w_{\mathrm{g}}=\sigma^{-2}\left(I_{\mathrm{g}}^{\circ}\right)$ is the weight of the observed intensity, $N_{\mathrm{R}}$ and $N_{\mathrm{P}}$ are the number of independent reflections and refined parameters, respectively.

\section{Previous results and discussion}

The structure of $\mathrm{Ni}_{3} \mathrm{Si}_{2}$ was obtained by Pilström in 1961 [11] by single crystal X-ray diffraction. The space group was determined to be $C m c 2_{1}$. The crystallographic parameters obtained by electron diffraction are shown in Table I together with the parameters from [11]. The space group was identified through the diffraction pattern in special directions $0 k l$ and $h k 0$ to be either $C m c m$ or $C m c 2_{1}$. The model was initially solved in the centrosymmetric space group, and then it was transformed to the noncentrosymmetric $C m c 2_{1}$ in order to compare with the published structure.

TABLE I

Unit cell parameters from electron diffraction (this work) and from X-ray single crystal data [11].

\begin{tabular}{c|c|c}
\hline \hline Parameter & EDT + PED & Literature [11] \\
\hline$\lambda[\AA]$ & 0.0335 & 0.71069 \\
crystal system & orthorhombic & orthorhombic \\
a $[\AA]$ & 12.5207 & 12.229 \\
$\mathrm{~b}[\AA]$ & 11.0491 & 10.805 \\
$\mathrm{c}[\AA]$ & 7.0209 & 6.924 \\
$V_{\mathrm{UC}}\left[\AA^{3}\right]$ & 971.2886 & 914.8982 \\
$Z$ & 16 & 16 \\
density $\left[\mathrm{g} \mathrm{cm}^{-3}\right]$ & 6.3527 & 6.7421
\end{tabular}

The figures of merit for the structure refinements from the model in the centrosymmetric space group (Table II) are essentially the same as in the noncentrosymmetric (Table III), without significant decrease for the latter. This factor indicates that the structure is most likely centrosymmetric. 
TABLE II

Refinement figures of merit for the model in the space group $\mathrm{Cmcm}$.

\begin{tabular}{c|c|c}
\hline \hline \multirow{2}{*}{ Parameter } & \multicolumn{2}{|c}{ EDT + PED } \\
\cline { 2 - 3 } & Dyn & Kin \\
\hline observed reflections & 1650 & 515 \\
refined parameters & 131 & 28 \\
$R$ (obs) [\%] & 6.80 & 21.73 \\
$R$ (all) [\%] & 10.10 & 23.13 \\
$w R$ (obs) [\%] & 6.46 & 24.78 \\
$w R$ (all) [\%] & 6.70 & 24.80 \\
GOF(obs) [\%] & 2.55 & 14.47 \\
GOF(all) [\%] & 2.13 & 14.00
\end{tabular}

TABLE III

Crystallographic parameters for the model in the space group $C m c 2_{1}$ for kinematical and dynamical refinements, and for the published structure [11]

\begin{tabular}{c|c|c|c}
\hline \hline Parameter & \multicolumn{2}{|c|}{ EDT + PED } & \multirow{2}{*}{ Literature [11] } \\
\hline Refinement & Dyn & Kin & \\
\hline observed reflections & 1650 & 1016 & 367 \\
refined parameters & 147 & 44 & \\
$R$ (obs) [\%] & 6.80 & 19.23 & \\
$R$ (all) [\%] & 10.10 & 21.31 & 11.8 \\
$w R$ (obs) [\%] & 6.44 & 20.68 & \\
$w R($ all) [\%] & 6.68 & 20.76 & \\
GOF(obs) [\%] & 2.55 & 10.72 & \\
GOF(all) [\%] & 2.13 & 10.26 &
\end{tabular}

The atomic coordinates were also analyzed in order to verify the symmetry of the model. When the model is transformed to lower symmetry space group $C m c 2_{1}, 3$ atoms split positions (Fig. 3). All the three atoms have small deviation from the symmetric position. As an example, atom Ni5, which is in the center of symmetry in the centrosymmetric space group, is shifted to atomic coordinates $(0.5,0.4981(6), 0.4983(9))$ in the noncentrosymmetric space group. These small displacements suggest that the correct space group is in fact $\mathrm{Cm} \mathrm{cm}$.

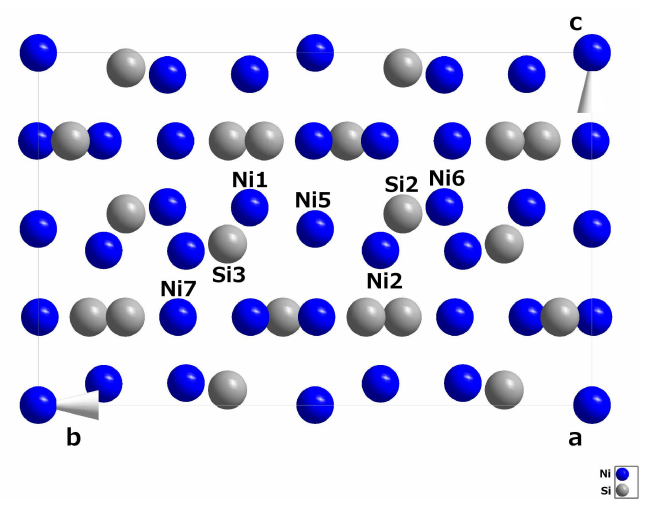

Fig. 3. Unit cell along [100] from the model in the noncentrosymmetric space group.
As expected, the dynamical refinement results in significantly lower figures of merit (Tables II, III), which confirm its superiority to the kinematical refinement.

\section{Conclusion}

It has been shown that reliable structure solution of nanocrystals can be obtained with the combination of electron diffraction tomography and precession electron diffraction. This combination gives three-dimensional diffraction information of the nanocrystal and suppresses the effects of dynamical interactions. However, the use of the dynamical theory of diffraction gives accurate structure refinement and lower figures of merit. The known structure of a nanowire of $\mathrm{Ni}_{3} \mathrm{Si}_{2}$ was analyzed using EDT and PED data. The kinematical and dynamical refinements were performed, showing the improvement in the accuracy when the dynamical theory was considered. The space group of the structure was determined as centrosymmetric, in disagreement with the published structure.

\section{Acknowledgments}

This work was supported by the Czech Science Foundation Project No. 13-25747S.

\section{References}

[1] U. Kolb, T. Gorelik, C. Kübel, M.T. Otten, D. Hubert, Ultramicroscopy 107, 507 (2007).

[2] U. Kolb, T. Gorelik, M. Otten, Ultramicroscopy 108 763 (2008).

[3] R. Vincent, P. Midgley, Ultramicroscopy 53, 271 (1994).

[4] J. Spence, Acta Crystallogr. A49, 231 (1993).

[5] L. Palatinus, J. Damien, P. Cuvillier, M. Klementová, W. Shinkler, L. Marks, Acta Crystallogr. A69, 171 (2013).

[6] V. Petříček, M. Dušek, L. Palatinus, The Crystallographic Computing System JANA2006, 2006.

[7] V. Petříček, M. Dušek, L. Palatinus, Z. Kristallogr. 229, 345 (2014).

[8] A. Reader, A. van Ommen, P. Weijs, R. Wolters, D. Oostra, Rep. Prog. Phys. 56, 1397 (1992).

[9] L. Palatinus, PETS - program for analysis of electron diffraction data, 2011.

[10] L. Palatinus, G. Chapuis, J. Appl. Crystallogr. 40, 786 (2007).

[11] G. Pilström, Acta Chem. Scand. 15, 893 (1961). 